\title{
Automated test generation technique for aspectual features in AspectJ
}

\begin{abstract}
Context: Aspect-oriented programming (AOP) has been promoted as a means for handling the modularization of software systems by raising the abstraction level and reducing the scattering and tangling of crosscutting concerns. Studies from literature have shown the usefulness and application of AOP across various fields of research and domains. Despite this, research shows that AOP is currently used in a cautious way due to its natural impact on testability and maintainability.
\end{abstract}

Objective: To realize the benefits of AOP and to increase its adoption, aspects developed using AOP should be subjected to automated testing. Automated testing, as one of the most pressing needs of the software industry to reduce both effort and costs in assuring correctness, is a delicate issue in testing aspect-oriented programs that still requires advancement and has a way to go before maturity.

Method: Previous attempts and studies in automated test generation process for aspectoriented programs have been very limited. This paper proposes a rigorous automated test generation technique, called RAMBUTANS, with its tool support based on guided random testing for the AspectJ programs.

Results: The paper reports the results of a thorough empirical study of 9 AspectJ benchmark programs, including non-trivial and larger software, by means of mutation analysis to compare RAMBUTANS and the four existing automated AOP testing approaches for testing aspects in terms of fault detection effectiveness and test effort efficiency. The results of the experiment and statistical tests supplemented by effect size measures presented evidence of the effectiveness and efficiency of the proposed technique at $99 \%$ confidence level (i.e. $\mathrm{p}<$ $0.01)$.

Conclusion: The study showed that the resulting randomized tests were reasonably good for AOP testing, thus the proposed technique could be worth using as an effective and efficient AOP-specific automated test generation technique.

Keyword: Software testing; Automated test generation; Testing tool; AspectJ; Empirical study 